\title{
Cost Consideration for Application Specific Microsystems' Physical Design Stages
} A new approach for microtechnological process design

\author{
R. Brück, A. Priebe, K. Hahn \\ University of Siegen, Institute of Computer Structures \\ Hölderlinstr. 3, D-57068 Siegen, Germany \\ Tel. +492717402472 , Fax. +492717402473 \\ email:hahn@rs.uni-siegen.de
}

Key words: microsystem physical design, process configuration, process optimisation, design verification, component technology, INTERNET tool access, JAVA

Abstract: $\quad$ The microsystem technology (MST) industry is characterised by small and medium sized enterprises (SMEs) specialised on products for application specific solutions rather than standardised mass volume production. Domains like medicine, automotive sensor technology, etc. are well-known. In this area of business the technology driven design approach known from micro electronics is not appropriate. Instead each design problem aims at its own, specific technology to be used for the solution. The variety of technologies nowadays in use, like Si-surface, Si-bulk, LIGA, laser, precision engineering requires a huge amount of expertise and support for choosing initially the appropriate fabrication technology for specific design requirements. This paper describes methods and tools that support the design of consistent process step configurations that allow to predict in advance the effort assigned to specific process arrangements. Based on formal process description means the microsystems' designer can edit, assess and optimize consistent technology step sequences with regard to costs, time, yield and feasibility providing an interface to later layout verification. The tools presented became recently accessible via the Internet using JavaBeans componentware. 


\section{MICROSYSTEM PHYSICAL DESIGN VS. MICROELECTRONICS DESIGN}

MEMS (so called in the US) or microsystems (so called in Europe) are able to provide the important interfaces between real world parameters and microelectronical information processing. Microstructured components contribute a substantial added value in many innovative products such as medical equipment, home office applications or automobiles.

As a result of some specific properties, the physical design process in microstructure technologies is not sufficiently supported. The design style is problem-oriented - as opposed to the technology oriented design style known from today's classical microelectronic systems design - which turns out to be the appropriate approach for microsystem design. Unlike in microelectronics the dependency between layout design and fabrication technology is very strong as many design properties (e.g. the size of structures in the third dimension) can only be realised choosing specific process parameters like materials, process steps or process resources.

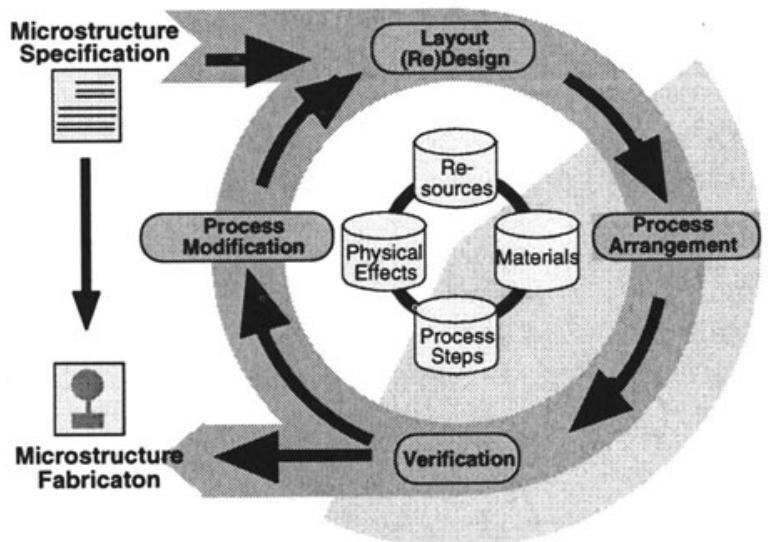

Fig 1. Circular Model of MEMS Design

In contrast to other disciplines of engineering the CAD tools used in this context at the moment were often developed for other purposes such as mechanical engineering or for IC layout design. Although efforts were made to enhance latter tools to deal with microsystem design constraints the application areas for these solutions are restricted. In general the methods and CAD systems available do often not meet the requirements of microsystem designers. [1] 
In the area of physical microsystem design that in lithography-based technologies is concerned with the design of mask layout geometry, the typical design cycle is dominated by the fact that the three-dimensional nature of the products calls for a particular sequence of processing steps and parameters to be specified for each design object. This results in the circle model for MEMS physical design. It is characterised by the following circular design flow as shown in figure 1.

Generating tasks creating the mask layout geometry for the design object and the specific process step sequence to be performed in order to generate the appropriate orthogonal extension of the design object during production. The process step specification part will be in the focus of this paper.

Checking tasks deriving a consistent set of design rules from the process sequence and applying it to the mask layout in order to find rule violations. If violations are detected, the design will have to be modified.

Modifying tasks used to determine what sort of changes will have to be made in order to turn the design into a correct version. This may include mask layout changes, process specification changes or changes in the higher level design.

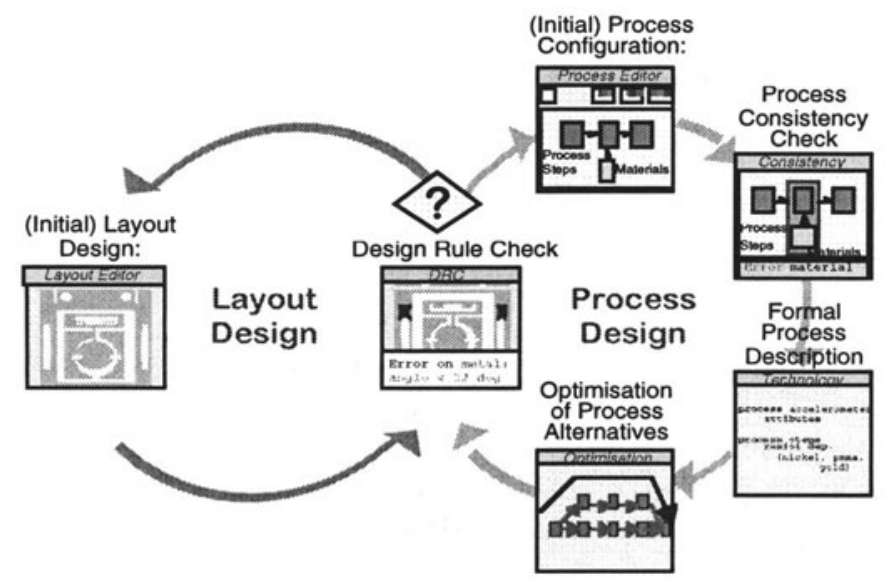

Fig. 2 Concurrent Design of Process and Layout

For more details of the circle model, see [2].

Figure 2 gives an alternative view of this model, showing the design flow as two concurrent, mutual dependent circles of layout and process design. The process editing tool to be presented shortly is used to support the process design cycle.

Each of the steps shown in this figure can be supported by specific design tools. Each of these tools is an independent software module, used to assist 
the user in a particular subtask of the whole design cycle. The degree of automation provided may differ substantially for each of the tools. The term assistants is used to denote this sort of independent software modules. The INTERLIDO-system presented in this paper thus can be regarded as a collection of weakly interacting assistants.

\section{INTERLIDO MICROSYSTEM DESIGN SYSTEM}

Based on these considerations the INTERLIDO MEMS design system has been developed at the Universities of Dortmund, Jena and Siegen. INTERLIDO supports both the design of appropriate process layouts for microstructure design as well as the construction of consistent and economically optimized process sequences. The link between process design tools and layout is based on a specific process description language LIDOPDL that allows the specification of all process characteristics that are relevant to layout design including constructs to define economic properties like cost and time of processes.

The system as a whole is composed of a set of assistants. It consists of a common user interface and a common technology and geometry database, both combined in the INTERLIDO-Manager. Figure 3 shows a system view of the INTERLIDO system. The user interface offers two major applications: LIDO-Pedit - the graphical process configuration editor and LIDO-Check. the microstructure design rule checker.

PEdit allows the configuration of process step sequences without requiring to compose textual LIDO-PDL descriptions. The process sequences can be checked with regard to their internal consistency and the system allows also the optimisation of fabrication processes towards least cost or time consumption. [3]

The consistent and optimised process sequence is used to automatically create a technology file in LIDO-PDL that might e.g. be used to derive geometrical design rules for the design verification module (Check) that checks the mask layout for rule violations. [4].

The system formerly developed in $\mathrm{C}++$ for workstation application, is completely re-implemented as componentware to be accessible via the Internet. The benefits of component based software with Internet access capabilities especially for MEMS design tools will be shown in the end section. 


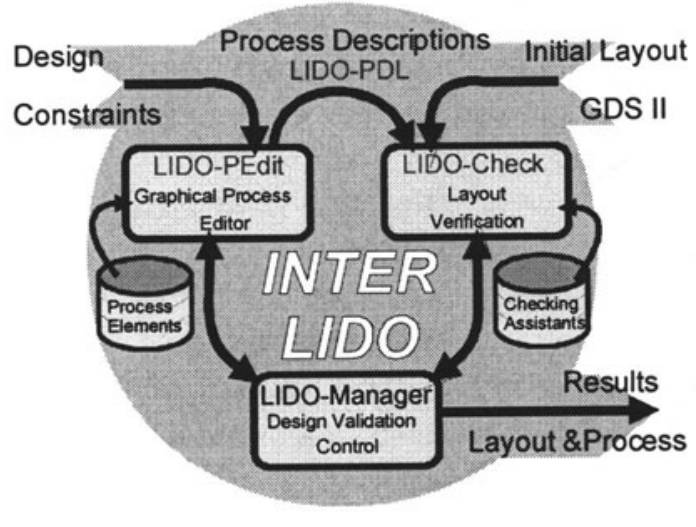

Fig. 3. INTERLIDO System View

\section{PROCESS DESCRIPTIONS}

The generation of formal description means for lithography-based micromachining processes was a precondition to realize the INTERLIDO system. The realization of the process description language LIDO-PDL is well documented in [5].

LIDO-PDL provides means to describe formally layout rules (with regard to later design verification steps) as well as process parameters. The basic idea for LIDO-PDL is the encapsulation of data (like process rules res. design rules) within objects and the configuration of these objects to complete process sequences. This approach reflects the microstructure physical design methodology with design specific process configurations. Different types of objects can be used. An excerpt of a LIDO-PDL process description is shown further down.

The complete process sequence is defined within a process object. A process consists of process steps carried out in a defined order.

Material objects, resource objects as well as layer objects can be assigned to process step objects. All types of objects can incorporate design rules and process parameters.

As a summary $L I D O-P D L$ provides language constructs for the description of

a) Process steps, materials, lithographic masks and useful assemblies of them as well as design rules to be associated with these object types

b) Process step networks taking into account process alternatives and process step concurrency. These process networks form the basis of process 
optimisations. Based on economical constraints associated with each process step an optimum utilisation of process resources can be determined making use of parallel process strands and the possibility to select among several alternatives.

c) Economic constraints for each object type. This includes process step execution time, process step resource utilisation, basic and marginal cost for process steps. The information can either be supplied as absolute values or as functions of process parameters like material selections, processing pressures and temperatures, process preparation or setup times and the like. This data is used to perform process optimisations with respect to economics considerations. [6]

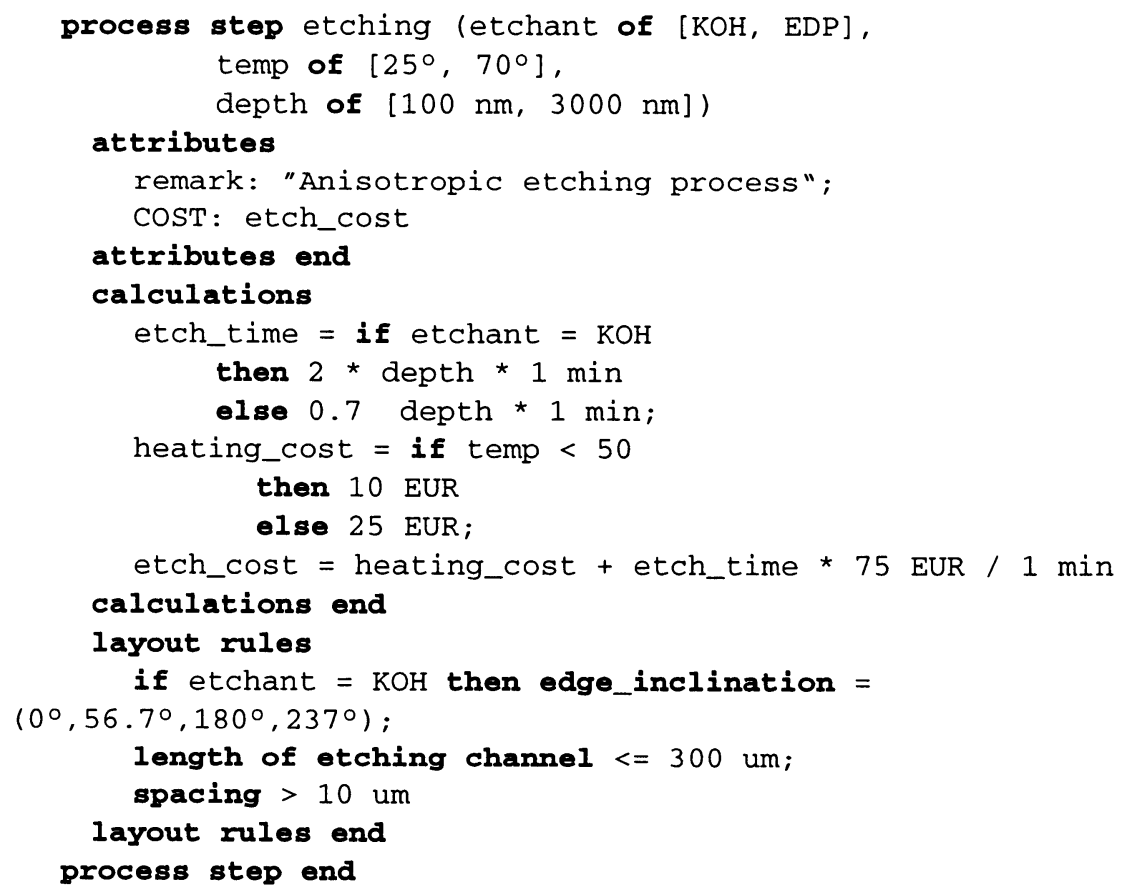

Once all parameters for the particular process are fixed the optimisation procedures of the LIDO-PDL compilation system will deliver the most costeffective path through this network.

\section{PEDIT PROCESS EDITING TOOL}

INTERLIDO-PEdit provides means to configure a process sequence according to the requirements and to the layout design of the intended microstructure and subsequently to check the current process configuration 
for consistency. The process arrangement is performed graphically in an editor window. The user selects design process elements like process steps, materials etc. from libraries based on the specific design task he/she is performing. These elements appear as icons that can be placed and graphically be connected to process sequences on the editor window. Each icon is related to process or technology information such as design rules or process configuration rules. Fig. 4 shows the assignment of process description data (that is transparent top the user) to the graphical icon representing process steps. [7]

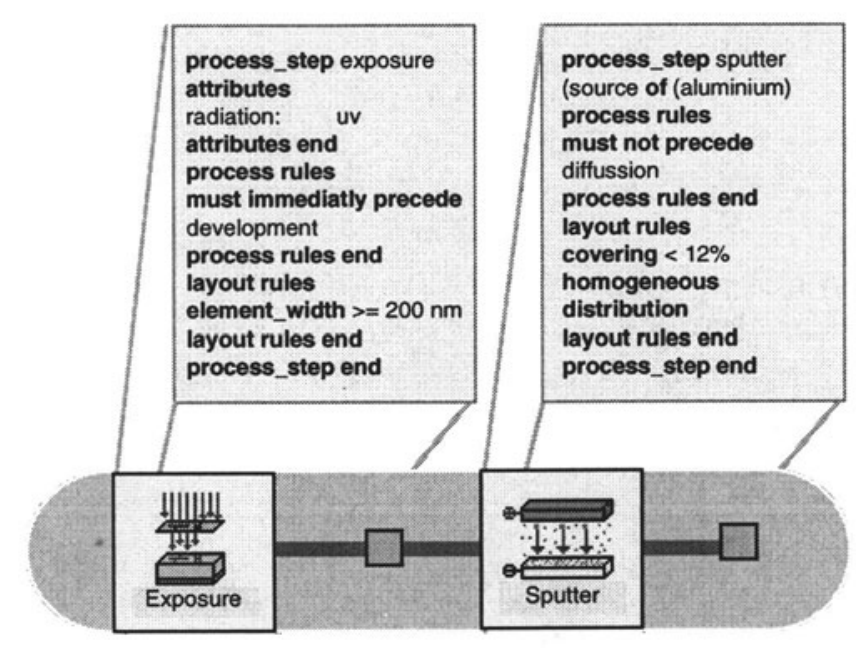

Fig. 4. Assignment of formal description entities to icons

\section{COST CONSIDERATION AND PROCESS OPTIMISATION}

As process networks with alternative process layouts (like shown in figure 5) can be represented in LIDO-PDL especially designed optimisation methods are implemented to find process sequences with minimum time or cost consumption. [8]

The graphically-based single-source-shortest-path algorithm analyses the process network and calculates the process configuration with lowest cost function values. The optimization module INTERLIDO-Opt fulfills the demands of designers wishing to learn about the economic implications of a process layout in early stages of the design flow. The optimisation requires the definition of variables as well as fixed costs within the appropriate 
LIDO-PDL objects. PDL offers constructs to declare all resource or process step related costs, time etc. Within the calculation parts of LIDO-PDL descriptions cost statements and function can be provided.

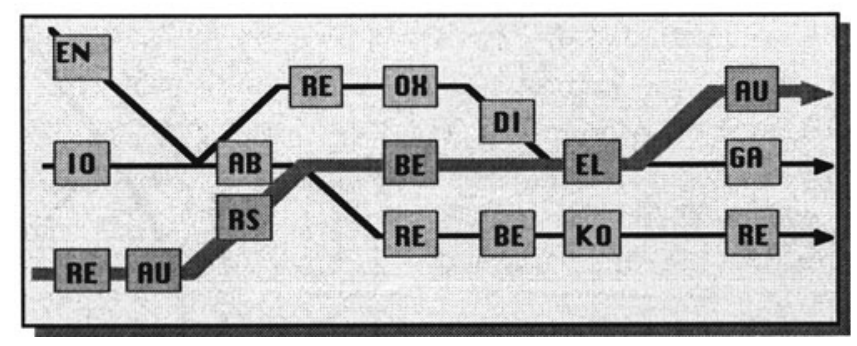

Fig. 5. Process network with optimum path

Based on this information INTERLIDO can pre-calculate the overall expenses assigned to specific process flows in case of alternative process forks. The optimum res. most cost-effective process step sequences can easily be determined before fabricating samples of the microstructures. Time, yield and cost statements can be used as objective functions for the optimisation task.

\section{NETWORK ACCESS BY COMPONENT TECHNOLOGY}

As has been pointed out in the previous chapters in micro engineering the design process has to be tailored towards every specific design object. As a consequence of this, specific design assistants have to be selected for each design task and especially adapted towards the restrictions to be met in each particular case. To achieve this, component-based software design is currently the method of the choice. It is targeted towards realizing so-called software components, executable pieces of software with a clearly defined interface, defined interoperability- and autonomy criteria, as well as the proof of reusability

Componentware is generally understood to be a collection of interacting components. Design assistants in INTERLIDO are realised as componentware based on the component technology JavaBeans by SUN Microsystems. JavaBeans is a platform independent component architecture for the Java Application Environment. An important precondition for a useful set of components is that they are well-adapted to each others. 


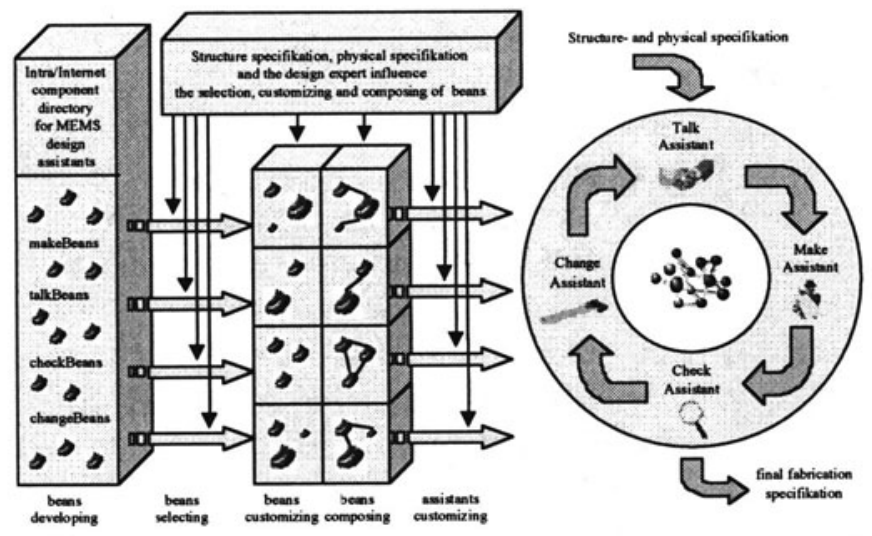

Fig. 6 Component based design assistants

Figure 6 shows an integrated view of the circle model for MEMS design. The Intranet/Internet component directory for physical MEMS design assistants contains various beans for the basic tasks to be performed during design.

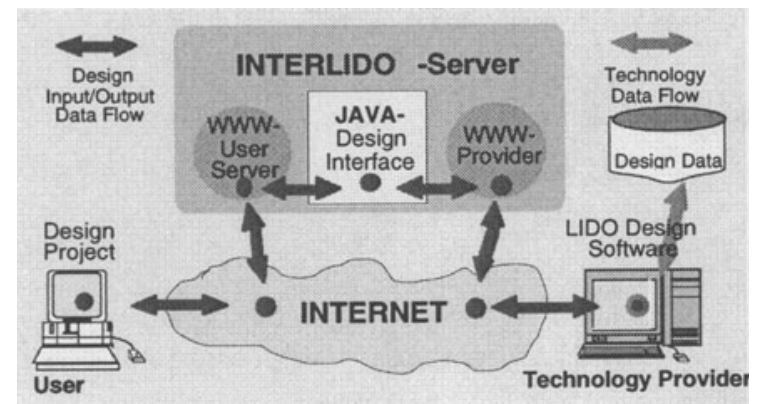

Fig 7: INTERLIDO-network software access

The INTERLIDO is a first prototype of a component-based MEMS physical design tool. At this point of time the dynamic creation of problemoriented beans has not yet been realised. INTERLIDO is currently used to demonstrate the robustness of a component approach and to show that it can really be useful and not prohibitively limited from a performance point of view, to provide tools that operate merely based on JAVA across the Internet. In addition not all of the LIDO components have been turned into components by now. LIDO-PEdit is the first part that is available based on 
this new technology. An overview of the INTERLIDO system has already been presented in Figure 3.

Here we only give some remarks on the process of using the INTERLIDO-system via the Internet. Figure 7 shows the principles of Internet-based software access, as they are realised in INTERLIDO. The INTERLIDO-Server is a broker system for user access to tools and technologies across the net. Technology providers will provide their design related knowledge in form of icons and LIDO-PDL descriptions. The LIDOPEdit functional components will be available on the INTERLIDO-Server and will access the technology related information via the net. The user interface is realized as a JAVA-applet to be run client side. [9]

The system presented so far is currently integrated into a training and working environment to be accessed via the Internet. The TRANSTEC project (No. MM1026) funded by the European Commission aims to implement a training course for different kinds of users teaching them how to take advantage and to solve problems using microsystem technologies.

\section{REFERENCES}

[1] Karam, J.M. (1998) Microsystems CAD, Proc. MME98 pp. 226-234, Ulvik, Norway

[2] Hahn, K., Brück, R. (1997) An Approach to Layout and Process Verification for Microsystem Physical Design. Journal Microsystem Technologies, pp.82-90

[3] Brück, R., Hahn, K. (1998) MEMS Process/Design and Optimization using Economical Constraints, Proc. MICRO SYSTEM Technologies 98, pp. 479-484

[4] Brück, R., Hahn, K., Reusch, B., (1998) Layout Verification Tools for MEMS Physical Design, Proc MSM 98, April 1998.

[5] Hahn, K., (1999) Methoden und Werkzeuge zur fertigungsnahen Entwurfsverifikation in der Mikrotechnik, Düsseldorf, VDI-Verlag

[6] Lettmann, F. (1997) Prozeßformalisierung zur Unterstützung von Wirtschaftlichkeitsaspekten beim Entwurf von Mikrosystemen. Diploma Thesis, Universität Dortmund

[7]Schneider, C.,Priebe, A., Brück, R., Schumer, C., (1999) Computer aided design tools for economical MEMS fabrication processes, Proc. DTM 99.

[8] Hahn, K., Brück, R., Priebe, A., Schneider, C. (1999) Cost Estimation for LIGA Fabrication Flows using Process Design Methods, Proc. HARMST99, Kisarazu, June 1999

[9] Brück, R., Schumer, C.(1999) Internet MEMS design tools based on component technology, Proc DTM 99, Paris,. 